\title{
Catalog of the Neotropical Masarinae (Hymenoptera, Vespidae)
}

\author{
James M. Carpenter ${ }^{1}$, Bolívar R. Garcete-Barrett ${ }^{2} \&$ Marcel G. Hermes $^{3}$
}

'Division of Invertebrate Zoology, American Museum of Natural History, Central Park West at $79^{\text {th }}$ Street, New York, NY 10024, U. S. A.
carpente@ amnh.org
${ }^{2}$ Museo Nacional de Historia Natural del Paraguay, Sucursal 1 Campus U.N.A., 2169 CDP, Central XI, San Lorenzo, Paraguay.
bolosphex@ sce.cnc.una.py
${ }^{3}$ Departamento de Zoologia, Universidade Federal do Paraná, CEP 81531 980, Cx. Postal 19020, Curitiba, Brazil. mghermes@gmail.com

\begin{abstract}
Catalog of the Neotropical Masarinae (Hymenoptera, Vespidae). A comprehensive catalog of the Neotropical Masarinae is presented. The lectotype of Trimeria howardi Bertoni, 1911, is designated.

KEYWORDS. Ceramiopsis; distribution; Gayella; Neotropical region; Paramasaris; pollen wasps; Trimeria.
\end{abstract}

RESUMO. Catálogo dos Masarinae Neotropicais (Hymenoptera, Vespidae). Um catálogo completo dos Masarinae Neotropicais é apresentado. O lectótipo de Trimeria howardi Bertoni, 1911 é designado.

PALAVRAS-CHAVE. Ceramiopsis; distribuição; Gayella; Paramasaris; região Neotropical; Trimeria; vespas-do-pólen.

The Neotropical fauna of pollen wasps is not rich in species, with 23 species described. But it is noteworthy for phylogenetic diversity, having one of the two tribes of Masarinae endemic, the Gayellini, with two genera, while the other tribe, Masarini, is represented by two endemic genera that are not each other's closest relative (Carpenter 1993). Most of the species were described during the last century, and there has been recent taxonomic work on each part of the fauna (Carpenter 1989, on Gayellini; Garcete-Barrett and Carpenter 2000, on the genus Ceramiopsis; and Carpenter 2001 and Hermes and Melo 2006, on Trimeria). There has also been recent work on the behavioral ecology of several species, and numerous floral records have been published. We bring together all of this literature here.

The arrangement of the catalog is alphabetical. Each species is followed by the original citation and then by synonyms and subsequent combinations, which are listed chronologically. Other references are listed chronologically under each synonym or generic change, regardless of changes in gender or incorrect spellings (these latter are indicated in brackets, with a exclamation mark for an incorrect spelling). To facilitate ready reference, each publication is cited in abbreviated form. Where two dates are listed, the first is the true date of publication, so far as can be determined, with the date following in parentheses being the date printed on the publication. The sex of the type specimens, if stated in the description, is then listed. The original locality is quoted for each description, and the type depository is then listed in parentheses. If the sex of the holotype or lectotype is known, this is listed with the depository, except where a single sex was specified in the original description, in which case this information is not repeated. A code word is used to designate most type depositories in the checklist, typically the name of city in which the collection is located; this precedes the name of the museum or institution in the list below. Museums or institutions where type specimens are kept are as follows.

Budapest: Hungarian Natural History Museum, Budapest, Hungary.

Buenos Aires: Museo Argentino de Ciencias Naturales "Bernardino Rivadavia," Buenos Aires, Argentina.

Cambridge: Museum of Comparative Zoology, Harvard University, Cambridge, Massachusetts, USA.

Curitiba: Coleção de Entomologia Pe. J. S. Moure, Departamento de Zoologia, Universidade Federal do Paraná, Brazil.

Genova: Museo Civico di Storia Naturale "Giacomo Doria," Genoa, Italy.

IPC: Instituto Pedagógico de Chile [Universidad de Chile, Santiago, Chile].

London: Natural History Museum, London, England.

Paris: Muséum National d'Histoire Naturelle, Paris, France.

San Francisco: California Academy of Sciences, San Francisco, California, USA.

San Lorenzo: Museo Nacional de Historia Natural del Paraguay, San Lorenzo, Paraguay.

Santiago: Museo Nacional de Historia Natural, Santiago, Chile.

Tucumán: Fundación y Instituto Miguel Lillo, Tucumán, Argentina.

Venezia: Museo Civico di Storia Naturale, Venice, Italy.

Washington: National Museum of Natural History, Smithsonian Institution, Washington, DC, USA.

Wien: Naturhistorisches Museum Wien, Vienna, Austria.

A summary of the distribution of each species follows the catalog, listing countries and regions/provinces/departments/ states. In the summaries, an asterisk denotes a new locality record. 
Tribe Gayellini Bradley, 1922

Genus Gayella Spinola

Gayella Spinola, 1851, in Gay, Hist. Fis. Polit. Chile, Zool., 6: 328, genus.

Type-species Gayella eumenoides Spinola, 1851, by monotypy.

Gazella Reed, 1894, Proc. Zool. Soc. Lond. 1893: 685, incorrect subsequent spelling of Gayella Spinola.

\section{araucana Willink}

Gayella araucana Willink, 1956, Bol. Acad. Nac. Cienc., Córdoba 39: 341, 342 (key), 346, figs. 3-4, female, male - "Chile, Prov. Santiago: Renca" (holotype female IPC); also from two other localities. - Richards, 1962, Revision. Study Masarid Wasps: 47 (key), fig. 39. - Willink, 1963, Acta Zool. Lilloana 19: 390 (Chile). - Giordani Soika, 1974, Boll. Mus. Civ. Stor. Nat. Venezia 25: 89 (key), 93, figs. 5, 7. - Willink and Ajmat de Toledo, 1979, Acta Zool. Lilloana 35: 429 (key), 431 (Chile), figs. 5, 11. Carpenter, 1989 (1988), Psyche 95: 231 (key), 234, figs. 3, 7, 16, 18, 28, 43, 48, 51, 58 (in eumenoides species group). - Pérez d'A, 1989, Acta Ent. Chil. 15: 277 (Chile; flower record: Homalocarpus dichotomus (Apiaceae)). - Willink and Chiappa, 1993, Acta Ent. Chil. 18: 120 (list). - Gess, 1996, Pollen Wasps: 37, 211, 305 (list). - Carpenter, 2001, Am. Mus. Novitat. 3325: 4 (list).

Distribution: CHILE: Atacama, Biobío, Coquimbo, Maule, O’Higgins, Santiago, Valparaíso.

\section{eumenoides Spinola}

Gayella eumenoides Spinola, 1851, in Gay, Hist. Fis. Polit. Chile, Zool. 6: 333, pl. II fig. 2, male, female - [Chile:] "provincias del norte y sobretodo en Santa Rosa" (lectotype female Paris). - de Saussure, 1852, Ét. Fam. Vesp. 1: 6, pl. III figs. 2a-d, pl. VIII figs. 4-4a. - Smith, 1857, Cat. Hym. Brit. Mus. 5: 8 (cat.). - de Saussure, 1875, Smithson. Misc. Coll. 254: 13. - Reed, 1893, An. Univ. Chile 84, Mem. Cient. Lit.: 878 (Chile); 1894, Proc. Zool. Soc. London 1893: 685. - Dalla Torre, 1894, Cat. Hym. 9: 8 (cat.). - Brèthes, 1903, An. Mus. Nac. Buenos Aires (3) 9 (2): 249 (Argentina). - Dalla Torre, 1904, Gen. Insect. 19: 13, pl. II figs. 3-3c (cat.). - von Schulthess, 1910, Dts. Ent. Z.: 189. Zavattari, 1912, Arch. Naturgesch. 78A (4): 7 (in subfamily Raphidoglossinae; probable syn. G. mutilloides de Saussure). - Jörgensen, 1912, An. Mus. Nac. Buenos Aires 22: 295. Bradley, 1922, Univ. Calif. Publ. Entomol. 1: fig. 97. - ClaudeJoseph (H. Janvier), 1930, An. Sci. Nat. Zool. (10) 13 (2): 350354, figs. 70-71 (Chile; nest; ethology; flower records: Schinus dependens (Anacardiaceae), Baccharis sp. (Asteraceae), Quillaja saponica (Rosaceae)). - Bequaert and Ruiz, 1942 (1940), Rev. Chilena Hist. Nat. 44: 215 (Chile). - Willink, 1956, Bol. Acad. Nac. Cienc., Córdoba 39: 342 (key), 344, figs. 1-2 (records from Argentina doubtful; Chile). - Giordani Soika, 1960 (1958), Boll. Mus. Civ. Stor. Nat. Venezia 11: 81 (key). - Richards, 1962, Revision. Study Masarid Wasps: 47 (key), fig. 42. Willink, 1963, Acta Zool. Lilloana 19: 390 (Chile). - Giordani Soika, 1974, Boll. Mus. Civ. Stor. Nat. Venezia 25: 90 (key), 98 (designation of lectotype), figs. 3-4, plate I (Chile). - Willink and Ajmat de Toledo, 1979, Acta Zool. Lilloana 35: 429 (key),
431 (Chile), figs. 3, 9. - Carpenter, 1989 (1988), Psyche 95: 231 (key), 234, figs. 1, 15, 27, 44, 52, 57 (in eumenoides species group). - Pérez d'A, 1989, Acta Ent. Chil. 15: 277 (Chile). Willink and Chiappa, 1993, Acta Ent. Chil. 18: 120 (list). - Gess, 1996, Pollen Wasps: 36, 66, 114, 212, 305 (list). - Carpenter, 2001, Am. Mus. Novitat. 3325: 4 (list).

Gayella sicheliana "de Saussure" von Schulthess, 1910, Dts. Ent. Z.: 189, as a synonym of G. eumenoides Spinola.

Distribution: CHILE: Atacama, Coquimbo, Maule, O’Higgins, Santiago, Valparaíso.

\section{luispenai Willink and Ajmat de Toledo}

Gayella luispenai Willink and Ajmat de Toledo, 1979, Acta Zool. Lilloana 35: 427, 428 (key), 429, figs. 1, 6-7, male, female "Rinconada, Jujuy, Argentina" (holotype male Tucumán); also from Mendoza; and Bolivia. - Carpenter, 1989 (1988), Psyche 95: 232 (key), 237, figs. 22, 45, 54, 63 (in mutilloides species group). - Gess, 1996, Pollen Wasps: 305 (list). - Carpenter, 2001, Am. Mus. Novitat. 3325: 4 (list).

Distribution: ARGENTINA: Jujuy, Mendoza; BOLIVIA: Potosí.

\section{mutilloides de Saussure}

Gayella mutilloides de Saussure, 1855, Ét. Fam. Vesp. 3: 114, female - "Le Chili" (London). - Smith, 1857, Cat. Hym. Brit. Mus. 5: 9 (cat.). - Dalla Torre, 1894, Cat. Hym. 9: 8 (cat.); 1904, Gen. Ins. 19: 13 (cat.). - von Schulthess, 1910, Dts. Ent. Z.: 189 (odyneroides [!]). - Zavattari, 1912, Arch. Naturgesch. 78A (4): 7 (probable syn. of Gayella eumenoides Spinola). - Bequaert, 1928, Ann. Mag. Nat. Hist. (10) 2: 145 (note on type). - Bequaert and Ruiz, 1942 (1940), Rev. Chilena Hist. Nat. 44: 216. - Willink, 1956, Bol. Acad. Nac. Cienc., Córdoba 39: 342 (key), 348, figs. 5-8 (Argentina). - Giordani Soika, 1960 (1958), Boll. Mus. Civ. Stor. Nat. Venezia 11: 82 (key), fig. 13. - Richards, 1962, Revision. Study Masarid Wasps: 47 (key). - Willink, 1963, Acta Zool. Lilloana 19: 389 (n. syn.: Gayella mutilloides nigerrima Giordani Soika). - Giordani Soika, 1974, Boll. Mus. Civ. Stor. Nat. Venezia 25: 90 (key), 95. - Willink and Ajmat de Toledo, 1979, Acta Zool. Lilloana 35: 428 (key), 431 (Chile), figs. 4, 10. Carpenter, 1989 (1988), Psyche 95: 231 (key), 237, figs. 14, 23, 61-62 (in mutilloides species group). - Pérez d'A, 1989, Acta Ent. Chil. 15: 279 (Chile). - Willink and Chiappa, 1993, Acta Ent. Chil. 18: 120 (list). - Gess, 1996, Pollen Wasps: 305 (list). Carpenter, 2001, Am. Mus. Novitat. 3325: 4 (list).

Gayella mutilloides nigerrima Giordani Soika, 1960 (1958), Boll. Mus. Civ. Stor. Nat. Venezia 11: 80, female - "Cile"(Venezia). - Willink, 1963, Acta Zool. Lilloana 19: 389 (n. syn. of G. mutilloides de Saussure).

Distribution: CHILE: Araucanía, Biobío, Santiago, Valparaíso; ARGENTINA: Chubut, Neuquén, Rio Negro.

\section{patagonica Willink}

Gayella patagonica Willink, 1956, Bol. Acad. Nac. Cienc. 39, Córdoba: 341, 342 (key), 350, figs. 9-11, female, male "Esquel, Chubut, Argentina" (holotype female Tucumán); also from Rio Negro and "N. W. Patagonia (1000-3000 ft.)." - 
Richards, 1962, Revision. Study Masarid Wasps: 47 (key). Willink, 1963, Acta Zool. Lilloana 19: 389 (n. syn.: Gayella cerceroides Giordani Soika; Chile). - Giordani Soika, 1974, Boll. Mus. Civ. Stor. Nat. Venezia 25: 90 (key), 96, figs. 6, 8. - Willink and Ajmat de Toledo, 1979, Acta Zool. Lilloana 35: 428 (key), 431, figs. 2, 8. - Carpenter, 1989 (1988), Psyche 95: 232 (key), 237, figs. 53, 55, 60 (in mutilloides species group). - Pérez d'A, 1989, Acta Ent. Chil. 15: 279. - Willink and Chiappa, 1993, Acta Ent. Chil. 18: 120 (list). - Gess, 1996, Pollen Wasps: 305 (list). Carpenter, 2001, Am. Mus. Novitat. 3325: 4 (list).

Gayella cerceroides Giordani Soika, 1960 (1958), Boll. Mus. Civ. Stor. Nat. Venezia 11: 82, female - "N. W. Patagonia: 10003000 piedi” (London). - Willink, 1963, Acta Zool. Lilloana 19: 389 (n. syn. of G. patagonica Willink).

Distribution: CHILE: Santiago; ARGENTINA: Chubut, Mendoza, Neuquén*, Rio Negro.

reedi Willink

Gayella reedi Willink, 1963, Acta Zool. Lilloana 19: 385, fig., female - "Casa Blanca, Valparaíso, Chile" (San Francisco). - Giordani Soika, 1974, Boll. Mus. Civ. Stor. Nat. Venezia 25: 90 (key), 99, fig. 9. - Willink and Ajmat de Toledo, 1979, Acta Zool. Lilloana 35: 429 (key), 431 (Chile), fig. 12. - Carpenter, 1989 (1988), Psyche 95: 231 (key), 235, figs. 17, 36, 49-50, 59 (male; in mutilloides species group)). - Pérez d'A, 1989, Acta Ent. Chil. 15: 279 (Chile; flower record: Adesmia melanthes (Papilionaceae)). - Willink and Chiappa, 1993, Acta Ent. Chil. 18: 120 (list). - Gess, 1996, Pollen Wasps: 37, 212, 305 (list). Carpenter, 2001, Am. Mus. Novitat. 3325: 4 (list).

Distribution: CHILE: Atacama, Coquimbo, Valparaíso.

\section{Genus Paramasaris Cameron}

Paramasaris Cameron, 1901, Trans. Am. Ent. Soc. 27: 311, genus. Type-species Paramasaris fuscipennis Cameron, 1901, by monotypy. Zethoides Cameron, 1904, Trans. Am. Ent. Soc. 30: 93, genus, non Zethoides Fox, 1899. - Bequaert, 1928, Ann. Mag. Nat. Hist. (10) 2: 144 (n. syn. of Paramasaris).

Type-species Zethoides flavolineatus Cameron, 1904, by monotypy.

Plesiozethus Cameron, 1905, Entomologist 38: 269, replacement name for Zethoides Cameron.

Metazethoides Schulz, 1906, Spolia Hym.: 213, unnecessary replacement name for Zethoides Cameron.

Paragayella Giordani Soika, 1974, Boll. Mus. Civ. Stor. Nat. Venezia 25: 87, 89, 99, genus. - Carpenter, 1989 (1988), Psyche 95: 227 (n. syn. of Paramasaris).

Type-species Paragayella richardsi Giordani Soika, 1974, by original designation and monotypy.

brasiliensis Giordani Soika

Paramasaris brasiliensis Giordani Soika, 1974, Boll. Mus. Civ. Stor. Nat. Venezia 25: 105, female, male - "Brasile: Nova Teutonia, Santa Catarina" (holotype female Cambridge). Carpenter, 1989 (1988), Psyche 95: 231 (key), 233, figs. 5, 12, 19, 25, 30, 35 (notes on type; Argentina). - Gess, 1996, Pollen Wasps: 305 (list). - Carpenter, 2001, Am. Mus. Novitat. 3325: 5 (list) - Hermes and Köhler, 2004, Cad. Pesq. Sér. Biol. 16 (2): 70 (key), 82, 100, fig. 1 (Brazil).
Distribution: Argentina:ARGENTINA: Misiones; BRAZIL: Rio Grande do Sul, Santa Catarina.

cupreus Giordani Soika

Paramasaris cupreus Giordani Soika, 1974, Boll. Mus. Civ. Stor. Nat. Venezia 25: 104, 106, female - "Columbia: Caqueta, Florencia, 480 m." (London). - Carpenter, 1989 (1988), Psyche 95: 231 (key), 233, figs. 13, 26, 34, 40, 47 (Colombia; Peru). Gess, 1996, Pollen Wasps: 305 (list). - Sarmiento-M., 1999, in Amat Garcia et al., Insect. Colombia II: 77. - Carpenter, 2001, Am. Mus. Novitat. 3325: 5 (list).

Distribution: PERU: Loreto; COLOMBIA: Caqueta, Magdalena, Putumayo.

\section{fuscipennis Cameron}

Paramasaris fuscipennis Cameron, 1901, Trans. Am. Ent. Soc. 27: 312, female - "Santa Fé Mountains, New Mexico" (London). - Dalla Torre, 1904, Gen. Ins. 19: 4 (cat.). - Bradley, 1922, Univ. Calif. Publ. Entomol. 1: 386 (in subfamily Euparagiinae; "I have not been able to learn of any mountains bearing this name”). - Bequaert, 1928, Ann. Mag. Nat. Hist. (10) 2: 143 (n. syn.: Zethoides flavolineatus Cameron; note on type; Guatemala). - Richards, 1962, Revision. Study Masarid Wasps: 48, figs. 17, 32-33, 43-46. - Giordani Soika, 1974, Boll. Mus. Civ. Stor. Nat. Venezia 25: 104, fig. 1. - Carpenter, 1989 (1988), Psyche 95: 231 (key), 233, figs. 21, 31, 33, 42, 56 (Mexico). - Gess, 1996, Pollen Wasps: 305 (list). - Sarmiento-M., 1999, in Amat Garcia et al., Insect. Colombia II: 77. - Carpenter, 2001, Am. Mus. Novitat. 3325: 5 (list).

Zethoides flavolineatus Cameron, 1904, Trans. Am. Ent. Soc. 30: 94, male - "Panama (Pacific side)" (London). - Bequaert, 1928, Ann. Mag. Nat. Hist. (10) 2: 143 (n. syn. of Paramasaris fuscipennis Cameron; note on types).

Plesiozethus flavolineatus; Cameron, 1905, Entomologist 38: 269; 1907, Entomologist 40: 62. - Zavattari, 1912, Arch. Naturgesch. 78A(2): 64, fig. (female; Colombia). - Bradley, 1922, Univ. Calif. Publ. Entomol. 1: 386 (in subfamily Euparagiinae).

Metazethoides flavolineatus; Schulz, 1906, Spolia Hym.: 213.

Distribution: COLOMBIA; PANAMA; GUATEMALA; MEXICO: Jalisco, Sinaloa.

richardsi (Giordani Soika)

Paragayella richardsi Giordani Soika, 1974, Boll. Mus. Civ. Stor. Nat. Venezia 25: 88, 101, fig. 2, pl. II, female - "Brasile: Mato Grosso, Serra Roncados [sic, Roncador], R. S. Base Camp" (London).

Paramasaris richardsi; Carpenter, 1989 (1988), Psyche 95: 231 (key), 232, figs. 6, 11, 20, 24, 29, 41, 46 (notes on type; Brazil). - Gess, 1996, Pollen Wasps: 305 (list). - Garcete-Barrett, 2001, Bol. Mus. Nac. Hist. Nat. Parag. 13: 41-45 (male; Paraguay; flower record: Hyptis brevipes (Lamiaceae)). - Carpenter, 2001, Am. Mus. Novitat. 3325: 5 (list).

Distribution: BRAZIL: Goias, Mato Grosso; PARAGUAY: Concepción*, Cordillera*, Paraguarí. 
Tribe Masarini Latreille, 1802 Subtibe Masarina

Genus Ceramiopsis Zavattari

Ceramiopsis Zavattari, 1910, Ann. Mus. Civ. Stor. Nat. Genova 44: 531, genus.

Type-species Ceramiopsis gestroi Zavattari, 1910, by monotypy.

gestroi Zavattari

Ceramiopsis gestroi Zavattari, 1910, Ann. Mus. Civ. Stor. Nat. Genova 44: 533, figs. A, B, female - [Brazil:] "Urucú, Corumbá (Matto Grosso)" (Genova); 1912, Arch. Naturgesch. 78A (2): 61, fig. - Bradley, 1922, Univ. Calif. Publ. Entomol. 1: 393. - Richards, 1962, Revision. Study Masarid Wasps: 79, figs. 79-87 (male; Argentina, Bolivia; probable syn.: Ceramiopsis paraguayensis Bertoni). - Gess, 1996, Pollen Wasps: 89, 115, 309 (list). - Garcete-Barrett and Carpenter, 2000, J. New York Ent. Soc. 108: 182 (key), 183, figs. 1-2 (redescription; Bolivia; Brazil; Paraguay; Argentina; flower records: Pontederia cordata (L.) and Eichornia azurea (Sw.) (Pontederiaceae)). - Carpenter, 2001, Am. Mus. Novitat. 3325: 13 (list).

Distribution: ARGENTINA: Entre Rios, Formosa, Santa Fé; PARAGUAY: Concepción*, Guairá, Paraguarí, San Pedro; BOLIVIA: Santa Cruz; BRAZIL: Mato Grosso do Sul.

\section{paraguayensis Bertoni}

Ceramiopsis paraguayensis Bertoni, 1921, Rev. Soc. Cient. Paraguay 1: 11, female, male - [Paraguay:] "Asunción, 'Quinta Caballero"' (lectotype female San Lorenzo) (seen entering burrow in ground). - Richards, 1962, Revision. Study Masarid Wasps: 79 (probable syn. of Ceramiopsis gestroi Zavattari). Gess, 1996, Pollen Wasps: 89, 309 (probable syn. of Ceramiopsis gestroi Zavattari). - Garcete-Barrett and Carpenter, 2000, J. New York Ent. Soc. 108: 182 (key), 184, figs. 3-4 (designation of lectotype; redescription; Bolivia; Paraguay; Argentina; flower records: Eichornia azurea (Sw.) and Eichornia crassipes (C. Martius) (Pontederiaceae)). Carpenter, 2001, Am. Mus. Novitat. 3325: 13 (list).

Distribution: ARGENTINA: Formosa; PARAGUAY: Alto Paraguay, Concepción*, Presidente Hayes, Central, Neembucú*, Paraguarí; BOLIVIA: Santa Cruz.

\section{Genus Trimeria de Saussure}

Trimeria de Saussure, 1854, Ét. Fam. Vesp. 3: pl. IV, fig. 2, genus [replacement name for Erynnis de Saussure].

Type-species: Erynnis americana de Saussure, 1853, by monotypy.

Erynnis de Saussure, 1853, Ann. Soc. Ent. Fr. (3) 1, Bull. II: xix, genus (2 species), non Erynnis Schrank, 1801 (Lepidoptera).

Type-species Erynnis americana de Saussure, 1853, by subsequent designation of de Saussure, 1855, Ét. Fam. Vesp. 3: 92.

Eurynnis Dalla Torre, 1894, Cat. Hym. 9: 4, incorrect subsequent spelling of Erynnis de Saussure.

Microtrimeria Bequaert, 1928, Psyche 35: 191, genus: 191. - van der Vecht and Carpenter, 1990, Zool. Verh. Leiden 260: 34 (n. syn. of Trimeria).

Type-species: Microtrimeria cockerelli Bequaert, 1928, by monotypy. Willinkia Richards, 1962, Revision. Study Masarid Wasps: 119, subgenus of Trimeria de Saussure. - van der Vecht and Carpenter, 1990, Zool. Verh. Leiden 260: 59 (n. syn. of Trimeria).

Type-species: "Trimeria buyssoni Brèthes, 1904 (as represented in coll. B. M.)," by original designation.

americana (de Saussure)

Masaris vespiformis Romand, 1851, Ann. Soc. Ent. France (2) 9, Bull.: lii [female not male; misidentification].

Erynnis americana de Saussure, 1853, Ann. Soc. Ent. France (3) 1, Bull. II: xx, female - "d'Amérique” (Paris).

Trimeria americana; de Saussure, 1854, Ét. Fam. Vesp. 3: pl. IV fig. 2; 1855, Ét. Fam. Vesp. 3: 81. - Smith, 1857, Cat. Hym. Brit. Mus. 5: 5 (cat.). - de Saussure, 1875, Smithson. Misc. Coll. 254: 4 (“Hab. Brazil"). - Dalla Torre, 1894, Cat. Hym. 9: 5 (cat.). - Brèthes, 1903, An. Mus. Nac. Buenos Aires (3) 9 (2): 374, fig. 4 (compared to T. buyssoni [=rhachiphora]). - Dalla Torre, 1904, Gen. Ins. 19: 7 (cat.). - du Buysson, 1910, Zool. Jahrb. Abt. Syst. 49: 241 (key). - Bertoni, 1911, An. Mus. Nac. Buenos Aires (3) 22: 104 (compared to T. howardi). - Zavattari, 1912, Arch. Naturgesch. 78A (2): 59 (key; type is from "Brasilien: Campos Geraes"). - Bertoni, 1921, Rev. Soc. Cient. Paraguay 1: 11 (Paraguay; flower record: Eryngium sp.). - Bradley, 1922 , Univ. Calif. Publ. Entomol. 1: 434 (list). - Richards, 1962, Revision. Study Masarid Wasps: 120 (key), 122 (notes on type). - Neff and Simpson, 1985, J. Kansas Entomol. Soc. 58 (4): 732 (Argentina; flower record: Heliotopium vernonifolium (Boraginaceae)). - Gess, 1996, Pollen Wasps: 212, 315 (list). Carpenter, 2001, Am. Mus. Novitat. 3325: 29 (Brazil; list). Hermes and Melo, 2006, Zootaxa 1155: 62 (key).

Distribution: ARGENTINA: Catamarca, Misiones*; PARAGUAY: Amambay, Caaguazú*, San Pedro*; BRAZIL: Distrito Federal, Goiás, Minas Gerais*, Mato Grosso do Sul*, São Paulo, Paraná.

\section{atacama (Fritz)}

Microtrimeria atacama Fritz, 1968, Rev. Chil. Ent. 6: 69, female, male - "Estación Cachiyuyo, provincia de Atacama, Chile" (holotype female Santiago). - Pérez d'A, 1989, Acta Ent. Chil. 15: 279 (Chile; flower records: Argylia radiata (Bignoniaceae), Heliotropium sp. (Boraginaceae), Cristaria inconspicua (Malvaceae)). - Willink and Chiappa, 1993, Acta Ent. Chil. 18: 120 (list). - Gess, 1996, Pollen Wasps: 212, 311 (list).

Trimeria atacama; Carpenter, 2001, Am. Mus. Novitat. 3325: 30 (list).

Distribution: CHILE: Atacama, Coquimbo*.

\section{bequaerti Willink}

Trimeria bequaerti Willink, 1951, Rev. Soc. Cient. Argentina 15: 78, figs. 1-6, female - "Amaicha del Valle, provincia de Tucumán (2.000 ms, s. n. m.)” (Tucumán); also from Salta; 1959, Neotropica 5: 1 (male; Bolivia), 3 (compared to T. monrosi). - Richards, 1962, Revision. Study Masarid Wasps: 120 (key), 125. - Garcete-Barrett, 1999, An. Soc. Entomol. Paraguay 1: 23 (Paraguay; flower records: Portulaca cryptopetala, $P$. grandiflora (Portulaceae), Alternanthera ficoidea (Amaranthaceae), Croton bomplandianus (Euphorbiaceae) 
and Baccharis salicifolia (Asteraceae). - Gess, 1996, Pollen Wasps: 315 (list). - Carpenter, 2001, Am. Mus. Novitat. 3325: 30 (list).

Distribution: ARGENTINA: Chaco*, Entre Rios*, Salta, Tucumán; PARAGUAY: Alto Paraguay*, Boquerón, Presidente Hayes*; BOLIVIA.

cockerelli (Bequaert)

Microtrimeria cockerelli Bequaert, 1928, Psyche 35: 191, 192, female - "PERU: Payta" (Cambridge). - Richards, 1962, Revision. Study Masarid Wasps: 121. - Gess, 1996, Pollen Wasps: 311 (list).

Trimeria cockerelli; Carpenter, 2001, Am. Mus. Novitat. 3325: 30 (list).

Distribution: PERU: La Libertad*, Piura.

\section{howardi Bertoni}

Trimeria howardi Bertoni, 1911, An. Mus. Nac. Buenos Aires (3) 22: 104, female, male - [Paraguay:] "Puerto Bertoni" (lectotype female Washington, here designated) (flower record: Talinum patens (Portulaceae)); 1918, An. Cient. Parag. (2) 3: 208 (list); 1921, Rev. Soc. Cient. Paraguay 1: 11. - Bradley, 1922, Univ. Calif. Publ. Entomol. 1: 434 (list). - Willink, 1951, Rev. Soc. Cient. Argentina 15: 77 (Argentina); 1959, Neotropica 5: 4 (compared to T. monrosi). - Richards, 1962, Revision. Study Masarid Wasps: 120 (key), 122 (Paraguay; Argentina), fig. 23. - Zucchi et al., 1976, Insect. Matsum. (N. Ser.) 8: 47-57, figs. 114 (nest; larva; ethology; Brazil). - Gess, 1996, Pollen Wasps: 56, 57, 58, 62, 64, 89, 90, 114, 116, 213 (flower records), 315 (list). - Mechi, 1999, Naturalia 24: 75-90 (nest; ethology); 1999, Rev. Biol. Tropic. 47: 938-948 (ethology; flower records: Bidens pilosa, Xanthium spinosum (Asteraceae), Stylosanthes guianensis and Zornia diphylla (Fabaceae)). - Carpenter, 2001, Am. Mus. Novitat. 3325: 30 (Brazil; list). - Hermes and Melo, 2006, Zootaxa 1155: 62 (key).

Distribution: ARGENTINA: Córdoba, Corrientes*, Entre Rios*, Misiones, Salta*, Santa Fé, Tucumán; PARAGUAY: Amambay*, Alto Paraná, Paraguarí*; URUGUAY*: Florida; BRAZIL: Goiás*, Mato Grosso do Sul*, Minas Gerais, Paraná*, Rio Grande do Sul, São Paulo.

Note: In order to provide an objective standard of reference for the application of the name, we are designating the lectotype, as follows. Lectotype female labeled "Puerto Bertoni/Alto Paraná/Paraguay/8.XII.1908" and "2602" and "Trimeria/howardi/W. Bertoni." A lectotype label has also been attached. The specimen is lacking the left midleg. We have seen several other syntypes, from San Lorenzo, London, and Washington, but the lectotype is in better condition than most other specimens. Although the original description did not mention the U. S. National Museum of Natural History as a depository, the label data are identical to other syntypes, and Bertoni is known to have exchanged specimens with the U. S. National Museum at a later time.

joergenseni Schrottky

Trimeria joergenseni Schrottky, 1909, Rev. Mus. La Plata
16: 137, female - “Argentina (Mendoza)" (lost). - Brèthes, 1910, An. Mus. Nac. Buenos Aires (3) 20: 285 (male; Argentina). Jörgensen, 1912, An. Mus. Nac. Hist. Nat. Buenos Aires (3) 15:294 (Argentina). - Zavattari, 1912, Arch. Naturgesch. 78A (2): 59 (key). - Bradley, 1922, Univ. Calif. Publ. Entomol. 1: 434 (list). - Willink, 1951, Rev. Soc. Cient. Argentina 15: 77 (Argentina). - Richards, 1962, Revision. Study Masarid Wasps: 120 (key), 126 (in subgenus Willinkia; Argentina). - Gess, 1996, Pollen Wasps: 315 (list). - Carpenter, 2001, Am. Mus. Novitat. 3325: 30 (Argentina; list).

Distribution: ARGENTINA: Córdoba*, Mendoza, Río Negro, San Luis, Santa Fé.

Note: Schrottky's collection was destroyed, however the identity of the species is not in doubt, and there are thus no grounds for designating a neotype under the present edition of the International Code of Zoological Nomenclature.

\section{monrosi Willink}

Trimeria monrosi Willink, 1959, Neotropica 5: 2, female [Argentina:] "Tucumán, Las Cejas" (Tucumán). - Richards, 1962, Revision. Study Masarid Wasps: 120 (key), 124 (redescription of type). - Neff and Simpson, 1985, J. Kansas Entomol. Soc. 58 (4): 732 (Argentina; flower record: Asteraceae spp.). - Gess, 1996, Pollen Wasps: 213, 315 (list). - Carpenter, 2001, Am. Mus. Novitat. 3325: 30 (Argentina; Bolivia; list).

Distribution: ARGENTINA: Córdoba*, Tucumán, Salta, San Luis*, Santa Fé, Santiago del Estero; BOLIVIA: Santa Cruz.

\section{neotropica (Mocsáry)}

Jugurtia neotropica Mocsáry, 1906, An. Hist.-Nat. Mus. Hung. 4: 197, male - "Paraguay: Asuncion" (Budapest).

Trimeria neotropica; du Buysson, 1910, Zool. Jahrb. Abt. Syst. 49: 241 (key; Paraguay). - Zavattari, 1912, Arch. Naturgesch. 78A (2): 59 (key), 60 (female; Paraguay). - Bertoni, 1918, An. Cient. Parag. (2) 3: 208 (list). - Bradley, 1922, Univ. Calif. Publ. Entomol. 1: 434 (list). - Willink, 1959, Neotropica 5: 4 (compared to T. monrosi). - Richards, 1962, Revision. Study Masarid Wasps: 120 (key), 122 (notes on type, redescription). - Gess, 1996, Pollen Wasps: 315 (list). - Carpenter, 2001, Am. Mus. Novitat. 3325: 30 ([error: Brazil; Argentina]; list).

Distribution: PARAGUAY: Caaguazú*, Central, Concepcion*, Cordillera, Itapúa*; ARGENTINA: Corrientes*, Entre Rios*.

\section{rhachiphora (Schletterer)}

Odynerus rhachiphorus Schletterer, 1891, Ent. Nachr. 17: 85, male - "Chile" (Wien).

Trimeria Buyssoni Brèthes, 1903, An. Mus. Nac. Buenos Aires (3) 9 (2): 371, 372, figs. 1-3, female - "Argentina: Catamarca" (Buenos Aires); also from Córdoba?; and Paraguay. - du Buysson, 1905, Bull. Soc. Ent. France 1905: 10 (male); 1910, Zool. Jahrb. Abt. Syst. 49: 241 (key). - Bertoni, 1911, An. Mus. Nac. Buenos Aires (3) 22: 104 (compared to T. howardi n. sp.). - Jörgensen, 1912, An. Mus. Nac. Hist. Nat. Buenos Aires (3) 15:294 (Argentina; flower record: Lippia nodiflora (Verbenaceae)). - Zavattari, 1912, Arch. Naturgesch. 78A(2): 
59 (key; Argentina). - Bertoni, 1918, An. Cient. Parag. (2) 3: 208 (list). - Bradley, 1922, Univ. Calif. Publ. Entomol. 1: 434 (list), figs. 23, 38-39, 53-55, 84, 105. - Bequaert, 1928, Psyche 35: 198. - Willink, 1951, Rev. Soc. Cient. Argentina 15: 77 (Argentina): 1959, Neotropica 5: 4 (compared to T. monrosi). - Richards, 1962, Revision. Study Masarid Wasps: 120 (key), 125 (Paraguay; Argentina), figs. 137-141 (in subgenus Willinkia). - Neff and Simpson, 1985, J. Kansas Entomol. Soc. 58 (4): 730732 (morphology; ethology; nest in burrow; Argentina; flower records: Heliotropum mendocinum (Boraginaceae), Sphaeralcea sp. (Malvaceae), Glandularia hookeriana, Lantana aristata, and Verbena sp. (Verbenaceae)). - Willink and Roig Alsina, 1993, J. Hym. Res. 2: 303 (n. syn. of $T$. rachiphorus [!]). - Gess, 1996, Pollen Wasps: 89, 115, 213, 315 (list). - Gess, 2004, J. Kansas Entomol. Soc. 77 (4): 709 (compared to Jugurtia namibicola).

Ancistroceroides rhachiphorus; Willink and Chiappa, 1993, Acta Ent. Chil. 18: 120 (list).

Trimeria rachiphorus [!]; Willink and Roig Alsina, 1993, J. Hym. Res. 2: 303 (n. syn.: Trimeria buyssoni Brèthes; Chile locality an error). - Garcete-Barrett, 1999, An. Soc. Entomol. Paraguay 1: 23 (rhachiphorus; Paraguay; flower records: Lippia sp. (Verbenaceae), Mikania cordifolia and Pluchea sagittalis (Asteraceae)). - Carpenter, 2001, Am. Mus. Novitat. 3325: 30 (rhachiphora; Uruguay; list).

Distribution ARGENTINA: Buenos Aires, Catamarca, Chaco*, Córdoba, Formosa, Jujuy, La Rioja*, Mendoza, Neuquén, Rio Negro*, Salta, San Juan, San Luis*, Santa Fé, Santiago del Estero, Tucumán; PARAGUAY: Boquerón, Central*, Itapuá, Presidente Hayes*; URUGUAY: Montevideo.

Note: There is a male specimen in the American Museum of Natural History with the locality label "CHILE, Nuble:/Los Robles/Nov. 30, 1983 L. Peña." In the absence of other specimens we prefer to regard this record as doubtful, either through mislabeling or as a stray. However, the species was described from Chile, and while since the recognition of the identity of the taxon by Willink and Roig Alsina (1993) this has been regarded as an error, it is possible that the original locality was correct.

robusta Hermes and Melo

Trimeria robusta Hermes and Melo, 2006, Zootaxa 1155: 61, 62 (key), 63, figs. 1-2, 4-7, 10, 13-15, female, male, nest "Brasil, Minas Gerais, 15 km sul da Jaíba" (holotype female Curitiba); also from Bahia (flower record: Waltheria (Sterculiaceae)).

Distr.: Brazil: Bahia, Minas Gerais.

rubra Hermes and Melo

Trimeria rubra Hermes and Melo, 2006, Zootaxa 1155: 61, 62 (key), 66, figs. 3, 8-9, 11-12, female - "Brazil, Rondônia ... Vilhena" (Curitiba).

Distr.: Brazil: Rondônia.

\section{REFERENCES}

Carpenter, J. M. 1989 (1988). The phylogenetic system of the Gayellini (Hymenoptera: Vespidae; Masarinae). Psyche 95: 211241.

Carpenter, J. M. 1993. Biogeographic patterns in the Vespidae (Hymenoptera): Two views of Africa and South America. In: P. Goldblatt (ed.). Biological Relationships between Africa and South America. Yale University Press, New Haven.

Carpenter, J. M. 2001. Checklist of species of the subfamily Masarinae (Hymenoptera: Vespidae). American Museum Novitates 3325: $1-39$.

Garcete-Barrett, B. R. \& J. M. Carpenter. 2000. A note on the taxonomy of the genus Ceramiopsis Zavattari (Hymenoptera: Vespidae; Masarinae). Journal of the New York Entomological Society 108: $181-186$.

Hermes, M. G. \& G. A. R. Melo. 2006. Two new species of Trimeria de Saussure from Brazil, with biological notes and a key to Brazilian taxa (Hymenoptera, Vespidae, Masarinae). Zootaxa 1155: 6168.

Willink, A. \& A. Roig-Alsina. 1993. On Odynerus rachiphorus Schletterer, a Masarinae (Trimeria), not a Eumeninae (Hymenoptera, Vespidae). Journal of Hymenoptera Research 2: 303 .

Received 22/11/2005; accepted 10/05/2006 